\title{
Coupling of Photoelectric and Triboelectric Effects as an Effective Approach for PZT- Based High-Performance Self-Powered Ultraviolet Photodetector
}

Li Su ${ }^{\# 1}$, Hua Yang Li $^{\# 1}$, Ying Wang ${ }^{1}$, Shuang Yang Kuang ${ }^{1}$, Zhong Lin Wang*, ${ }^{1,}$, Guang $Z h u^{* 1}$

1 Beijing Institute of Nanoenergy and Nanosystems, Chinese Academy of Sciences; National Center for Nanoscience and Technology (NCNST), Beijing, 100083, P. R. China

2 School of Materials Science and Engineering, Georgia Institute of Technology, Atlanta, GA 30332, USA.

\# Authors with equal contribution

* Correspondence: zlwang@binn.cas.cn, zhuguang@binn.cas.cn

Keywords: Lead zirconium titanate, Polarization, Self-powered, Triboelectric effect, UV photodetector

A high-performance self-powered UV photodetector (UV-SPPD) using a PZT film was fabricated and characterized. It has a dual sensing mechanism that relies on the joint properties of a photoelectric effect and a triboelectric effect on the surface of the ferroelectric material. The UV-SPPD exhibits excellent responsivity (15 mA W$\left.{ }^{-1}\right)$, large detectivity $\left(2 \times 10^{10} \mathrm{~cm} \mathrm{~Hz}^{-1 / 2} \mathrm{~W}^{-1}\right)$, a fast photo response time $(<50 \mathrm{~ms})$, and good stability and repeatability. This work creates new opportunities for designing high-performance selfpowered UV photodetectors and other tribotronic devices.

\section{Introduction}

Ultraviolet (UV) photodetectors are of critical importance in a variety of scientific and industrial applications, including communications[1], remote control[2], environmental monitoring[3], binary switches in imaging techniques[4], chemical/biological sensing[5], next-generation memory storage [6], and optoelectronic circuits [7]. A conventional UV 
photodetector normally has a sandwich-type multi-layered structure that consists of a photosensitive material and two electrodes [8, 9]. Recently, self-powered sensors have emerged as a new class of sensors that can operate without an electric power source [10]. These sensors can generate electric signals on their own by converting other types of energy into electricity. By measuring and analyzing the electrical signal that can be altered by a certain external stimulus, the presence and the magnitude of the stimulus can then be derived [11]. Such devices have been reported in the applications of detecting mechanical motions [12], chemical molecules [13], thermal agitations [14], and photo excitations [15]. Our group recently reported a $\mathrm{MAPbI}_{3}$-based self-powered photodetector that exhibited excellent responsivity $\left(7.5 \mathrm{~V} \mathrm{~W}^{-1}\right)$, rapid response time $(<80 \mathrm{~ms})$, great repeatability, and broad detection range from UV to visible light. Although the perovskite material has excellent light sensitivity and broad-band light absorption, its semiconducting property is detrimental to maintaining high-density triboelectric charges on its surface [16]. Therefore, the performance of self-powered photodetectors still has much room for improvement from a material aspect.

Ferroelectric materials are being studied as promising candidate materials for UV detection due to their attractive properties, such as switchable rectifying behavior [17], polarization-dependent photo-voltage [18], efficient electron-hole separation at ferroelectric domain walls [19], and ultra-fast carrier dynamics upon femtosecond optical excitation [20]. Specifically, lead zirconate titanate $\left(\mathrm{Pb}(\mathrm{Zr}, \mathrm{Ti}) \mathrm{O}_{3}\right)$ has received particular attention because of its large remnant polarization [21]. L. Pintilie et al. [22] reported a sandwich-structured UV photodetector based on a PZT thin film with a current responsivity as high as $1 \mathrm{~mA} \mathrm{~W} \mathrm{~W}^{-1}$. B. Chen et al. [23] developed a transparent PZT-based photodetector that could be well controlled using an externally applied electric field. In this work, we report a UV selfpowered photodetector (UV-SPPD) that relies on the joint properties of the photoelectric effect and the triboelectric effect of a PZT film. The UV-SPPD exhibits an excellent responsivity $\left(15 \mathrm{~mA} \mathrm{~W} \mathrm{~W}^{-1}\right)$ and detectivity $\left(2 \times 10^{10} \mathrm{~cm} \mathrm{~Hz}^{-1 / 2} \mathrm{~W}^{-1}\right)$, which are at least two 
orders-of-magnitude higher than the ferroelectric material-based UV photodetectors of previous reports $[24,25]$. The UV-SPPD also demonstrates a comparable response time $(<50$ ms) and good stability and repeatability. Considering the measurements from a scanning Kelvin probe microscope (SKPM) and a conductive atomic force microscope (CAFM), we propose a two-fold sensing mechanism in which both surface charge density and inner conductivity of the polarized PZT film are immediately altered upon UV illumination. Our results indicate the promise of ferroelectric materials for use in high-performance UV photodetectors.

\section{Results and Discussions}

\subsection{Device fabrication and materials characterization}

A UV-SPPD is composed of two separate parts, as illustrated by the diagram shown in Fig. 1a. The top and the bottom parts both have lateral dimensions of $15 \mathrm{~mm}$ by $15 \mathrm{~mm}$. In the bottom part, a PZT film of $\sim 80 \mu \mathrm{m}$ in thickness is fabricated onto a transparent FTO $\left(\mathrm{SnO}_{2}: \mathrm{F}\right)$ substrate. The top part is a copper film deposited onto an elastic buffer layer that aids effective surface contact between the copper and the PZT. The copper film and the FTO layer act as the upper and the bottom electrodes, respectively, which are connected to the positive and the negative terminals of an electrometer for data acquisition. The detailed fabrication process is presented in the Experimental Section. The surface morphology of the PZT surface is characterized using a scanning electron microscope (SEM) and atomic force microscope (AFM). The top view in Fig. 1b displays the polycrystalline feature of the PZT film with grain sizes of $\sim 8-10 \mu \mathrm{m}$. The inset shows a cross-sectional view that confirms the thickness of the PZT film as $\sim 80 \mu \mathrm{m}$. The x-ray diffraction pattern shown in Fig. 1c illustrates the single phase of perovskite for the PZT film. The AFM measurement results shown in Fig. 1d reveal an average surface roughness of $172.90 \mathrm{~nm}$. Fig. 1i presents the $P-E$ hysteresis curve of the PZT film, showing typical hard ferroelectric properties with a large remnant polarization as high as $2 \mathrm{P}_{\mathrm{r}}=60 \mu \mathrm{C} \mathrm{cm}^{-2}$ and $2 \mathrm{E}_{\mathrm{c}}=210 \mathrm{kV} \mathrm{cm}^{-1}$. As shown in the transmittance spectra (Fig. 
1f), a steep absorption edge appears at 340-450 nm. Fig. 1g shows that the bandgap of the PZT film is approximately $3.2 \mathrm{eV}$, which is in agreement with previous reports in the literature [26]. Upon externally applied mechanical agitation, the UV-SPPD generates electrical signals by converting mechanical energy into electricity that is then measured and analyzed. When the copper electrode repeatedly contacts the ferroelectric PZT film, an alternating short-circuit current $\left(I_{\mathrm{sc}}\right)$ is obtained (Fig. 1h). This electricity-generating process is enabled by the coupling effect of triboelectrification and electrostatic induction, based on the theory of the triboelectric generator, as previously reported in the literature [27-29].

\subsection{Detection results}

Here, three groups of UV-SPPDs are prepared. The first is based on PZT films that have no polarization; the other two groups have PZT films with opposite polarization directions. The group with the dipole moment pointing up is denoted as $P_{\text {up }}$, whereas the one with the reversed polarization is referred to as $P_{\text {down }}$. Without illumination, the $P_{\text {down }}$ samples generate an $I_{\mathrm{sc}}$ that has a peak-to-peak value of $3.7 \mu \mathrm{A}$, as shown in Fig. 2a. Once the UV-SPPD is exposed to UV illumination supplied by an $\mathrm{Hg}$ lamp (365 nm, $100 \mu \mathrm{Wcm}^{-2}$ ), the current amplitude substantially reduces in an immediate response. It was found that the current amplitude of the $P_{\text {down }}$ samples reduced by $90 \%$ under the UV illumination and by only $12 \%$ under full-sun illumination, as shown in Figs. 2a and 2b, respectively. Once the illumination is turned off, the current amplitude rapidly recovers to the original value. The difference between the UV illumination and the full-sun illumination is attributed to the absorption characteristics of the PZT film, which mainly absorbs UV light but not visible light (Fig. 1f).

For the $P_{\text {down }}$ samples, an exceptionally high ratio of $I_{\text {dark }}$ to $I_{\text {light }}$ enables them to serve as UV photodetectors. The major figures of merit for evaluating a photodetector are discussed as follows. Foremost, the responsivity $(R)$, which indicates the sensitivity of a photodetector in response to an optical stimulus, can be defined using the following equation: 
$R=\frac{\Delta I}{A \Delta P}$

where, $\Delta I$ is the variance of the current amplitude resulting from the increment of light intensity, $A$ is the illuminated area, and $\Delta P$ is the light intensity. The change of the current amplitude at different light intensities $\left(I_{\text {dark }}-I_{\text {light }}\right)$ is plotted in Fig. 2c, and the average responsivity can be obtained by linear fitting, which generates a value of $15 \mathrm{~mA} \mathrm{~W}^{-1}$, which represents a 15-to-100-fold enhancement compared to previously reported photodetectors based on ferroelectric materials. [24, 30, 31]

Second, the detectivity $\left(D^{*}\right)$ that embodies the detecting ability of a photodetector is given by the following equation:

$$
D^{*}=\frac{\sqrt{A} R}{\sqrt{2 q I_{\text {light }}}}
$$

Where, $R$ is the responsivity, $A$ is the illuminated area, $q$ is the unit charge, and $I_{\text {light }}$ is the amplitude of light current. In our case, the maximum detectivity of $2 \times 10^{10} \mathrm{~cm} \mathrm{~Hz}^{-1 / 2} \mathrm{~W}^{-1}$ is achieved, which is far superior than that of previous ferroelectric-material-based photodetectors by at least two orders of magnitude. [25, 31] The low amplitude of the light current and the relative large responsivity $(R)$ enables the UV-SPPD to obtain a high $D^{*}$.

Furthermore, the signals remains nearly unchanged after 15 cycles of operation (as shown in Fig. 2d), pointing to the excellent stability and reliability of the UV-SPPD. Although the accurate response time of the UV-SPPD cannot be derived due to the constantly changing current, it can still be approximately estimated. Fig. 2e shows the current sharply decreases by more than $90 \%$ within a half period of 50 ms. Therefore, the response time of the UV-SPPD is found to be less than $50 \mathrm{~ms}$, which is superior to the results of previous reports by Sharma et al. [32] and Zomorrodian et al. [33] Fig. 2f shows the photocurrent spectral response of the UV-SPPD at $254 \mathrm{~nm}, 365 \mathrm{~nm}$ and $400 \mathrm{~nm}$ with an incident intensity of $100 \mu \mathrm{W} \mathrm{cm}{ }^{-2}$, respectively. It was observed that the $\Delta I$ illuminated at $254 \mathrm{~nm}$ and $365 \mathrm{~nm}$ were almost the 
same $(3.2 \mu \mathrm{A})$, but greatly decreased to $0.5 \mu \mathrm{A}$ at $400 \mathrm{~nm}$. The result has a good agreement with the transmittance spectrum in Fig. 1(f).

For the systematization of the research, the samples without polarization and with $P_{\text {up }}$ were also measured to determine the UV light sensitivity. Without additional polarization, the $I_{\mathrm{sc}}$ reduced $50 \%$ upon UV illumination, as shown in Figs. $2 \mathrm{~g}$ and i. If the polarization is reversed, the $P_{\text {up }}$ samples produce only $20 \%$ of the current variation (Figs. $2 \mathrm{~h}$ and i). Thus, among the three groups of UV-SPPDs, the $P_{\text {down }}$ samples have the most significant sensitivity. In addition, the detection characteristics of the UV-SPPD are independent of the external exciting frequnency. As shown in Fig. 2j, as the frequency changes from 5 to $15 \mathrm{~Hz}$, the current upon UV illumination remains constant.

\subsection{Detection mechanism}

To explain the experimental results, we constructed the model depicted in Fig. 3. The current of the whole circuit can be divided into $I_{\text {total }}, I_{\mathrm{sc}}$ and $I_{\text {inner, }}$ which represent the total current generated by the circuit, the measured short-circuit output current, and the internal current of the electric source, respectively. The relationship between the three parts can be expressed using the following equation:

$$
I_{\text {total }}=I_{\mathrm{sc}}+I_{\text {inner }}
$$

Upon illumination, excitons are generated in the PZT layer. For the case without polarized PZT film, when $\mathrm{Cu}$ is in contact with PZT, the self-polarization causes the electrons conducted to the FTO electrode and the holes reaching to the surface to be neutralized by the negative triboelectric charge. The reduced surface charge density and enhancement of thinfilm conductivity result in a decrease of the $I_{\text {total }}$ but an increase of the $I_{\text {inner }}$. Thus, the measured output of the SPPD $I_{\mathrm{sc}}$ was found to be obviously decreased. When the PZT film was in the $P_{\text {down }}$ state, the strong built-in electrical field could separate the photo-generated electrons effectively, and more charges are driven to the electrodes. Thus, the varied surface charge density and thin-film conductivity is much higher than those of the case without 
polarization, causing a tremendous decrease in the $I_{\mathrm{sc}}$ output. However, the charge flowing direction was reversed in the case of the $P_{\text {up }}$ PZT film, indicating the invariant conductivity value but an increased $I_{\text {total }}$ and leading to a minimum reduced $I_{\mathrm{sc}}$ output.

\subsection{SKPM and CAFM measurement}

To explain the above-described model, we proposed a two-fold sensing mechanism based on the variation of the surface charge density and the conductivity. First, we used scanning Kevin probe microscopy (SKPM) at a bias voltage of $3 \mathrm{~V}$ to characterize the surface charge variation on the PZT film upon UV illumination (Fig. S1). The obtained surface potential from the SKPM measurement is a direct indicator of the surface charge density [34]. Initially, the original value of the surface potential is obtained as a reference point. As shown in Figs. 4a, 4e and 4i, the initial surface potential is found to be a minimum for all of the three groups of samples. Subsequently, the PZT surface was rubbed by an Ir-based tip with Pt coating in contact mode. The average surface potential of all of the three groups is found to be approximately $-600 \mathrm{mV}$, indicating the presence of negative charges on the PZT film as a result of triboelectrification from the rubbing of the metal tip. When the UV illumination is introduced after rubbing, the variation of the surface potential proves to be polarizationdependent. For the $P_{\text {down }}$ samples, the surface potential immediately rises from -610 to -398 $\mathrm{mV}$ (Figs. 4b and 4c) upon illumination. This change represents an instantaneous decrease of the surface charge density. For the PZT film without extra polarization, the surface potential barely changes, as shown in Figs. $4 \mathrm{f}$ and $4 \mathrm{~g}$. A very slight increment is likely attributed to the intrinsic polarization within in the PZT film, which will be further elaborated below. In contrast, the opposite change is observed in the $P_{\text {up }}$ samples. Shown in Figs. $4 \mathrm{j}$ and $4 \mathrm{k}$, the surface potential immediately decreases from -610 to $-792 \mathrm{mV}$ as the result of illumination, indicating the generation of more negative surface charges. The above results can be attributed to the photovoltaic and ferroelectric properties of the PZT film. The UV illumination generates a large number of electron-hole pairs within the PZT film. Once 
created, the electron-hole pairs are separated by an inner polarization filed. In the case of the $P_{\text {down }}$ samples, electrons drift to the top surface, neutralizing the negative triboelectric charges, as illustrated in Fig. 4d and Fig. 4h. However, it is the electrons that migrate to the surface in the case of $P_{\text {up }}$ samples, supplementing the already existing negative surface charges (Fig. 4i). Therefore, in terms of surface charge density, only the $P_{\text {down }}$ samples can contribute to the reduced $I_{\mathrm{sc}}$ when exposed to UV light. Subsequently, the surface charge density under UV illumination is found to remain almost unchanged regardless of the polarization direction (Figs. 4c, 4g and 4k). This observed long-time retention of the surface charge can be explained by the highly insulating property of the PZT material. [35]

Second, the conductivity of the ferroelectric PZT film is found to be another mechanism for UV detection. Here, conductive probe atom force microscopy (CAFM) in contact mode is used (Fig. S2) to characterize the conductivity. The UV illumination is directed onto the sample surface via an optical lens without a bias voltage. The measured current is indicative of the conductivity of the PZT film between the AFM tip and the FTO substrate. [36] For the $P_{\text {down }}$ sample, as the illumination is applied, the current flowing through the film shows a surge compared to that in darkness. The two-dimensional mapping of the current distribution is shown in Fig. 5a. The cross-sectional profile of the current distribution is quantitatively shown in Fig. 5b. The illuminated area has a photocurrent of 8.2 pA, which is nearly 9-fold higher than the dark current $(0.93 \mathrm{pA})$. A similar increase of the photocurrent is observed for the $P_{\text {up }}$ sample, as shown in Figs. 5c and 5f; however, the polarity is found to be opposite due to the reserved polarization direction. For the control sample without polarization, the generated photocurrent is very small, which is attributed to the weak intrinsic polarization as illustrated in Figs. 5b and 5e. Because of the enhanced conductivity, the inner resistance of the PZT film decreases. This decrease leads to a current that leaks through the PZT film. Therefore, the measured $I_{\mathrm{sc}}$ in the external circuit reduces once illumination is present.

\section{Conclusion}


In summary, we developed an integrated UV-SPPD using ferroelectric PZT film that demonstrates an excellent performance without reliance on an external power source. The performance parameters of the UV-SPPD are much better (two orders-of-magnitude improvement in responsivity and detectivity), comparable response time and good stability and repeatability. Based on the CAFM and SKPM measurements, the physical mechanism was interpreted to be an immediate alteration of the surface charge density and thin-film conductivity upon illumination. All the results demonstrated that this PZT-based SPPD is a great candidate for applications in UV light detection and may be extended to applications in other types of self-powered sensors as well as tribotronics.

\section{Experimental Section}

Synthesis and Characterization of Ferroelectric PZT Film: The detailed processing conditions to synthesize ceramic PZT are described elsewhere. After hot-pressing calcinations at $1300{ }^{\circ} \mathrm{C}$ under a pressure of $40 \mathrm{MPa}$, both sides of the sample were polished. The sample was then cut into a size of $15 \times 15 \times 0.1 \mathrm{~mm}^{3}$.

The surface, cross-section and crystal structure of the as-fabricated perovskite-based film were characterized using scanning electron microscopy (SEM, Hitachi, SU8020), atom force microscopy (AFM, MFP-3D SPM), and X-ray diffraction (XRD, X pert 3), respectively. A UV-Vis-NIR spectrophotometer (Shimadzu, UV-3600) was used to analyze the light absorption of the perovskite-based film. The polarization - electric field $(P-E)$ hysteresis loops of the PZT film were measured at $1 \mathrm{kHz}$ using a precision workstation ferroelectric tester (Radiant Technologies, Inc. NM, USA).

Fabrication of the Self-Powered Photodetectors: The self-powered photodetector was fabricated in two ways. In one approach, acrylic substrates with dimensions of $15 \mathrm{~mm}$ by 15 mm were prepared by laser cutting. A copper layer of $200 \mathrm{~nm}$ thickness was deposited by magnetron sputtering as the top triboelectric layer and the top electrode. In the other approach, the PZT films were constructed on FTO glass substrates, which had a dimensions of $15 \mathrm{~mm}$ 
by $15 \mathrm{~mm}$. In addition, we applied a high voltage of greater than $500 \mathrm{~V}$ across the PZT film for electric polarization at room temperature. Subsequently, two pieces of polyimide films with dimensions of $45 \mathrm{~mm}$ by $15 \mathrm{~mm}$ by $1.25 \mathrm{~mm}$ were prepared and attached to the substrates at opposite edges. These films served as braces that created a gap between the two contact surfaces. Finally, conducting wire was connected to each of the electrodes for measurement.

Measurements: Short-circuit current was measured using a Keithley 6514A electrometer under the illumination of an $\mathrm{Hg}$ lamp $\left(365 \mathrm{~nm}, \sim 100 \mu \mathrm{W} \mathrm{cm}{ }^{-2}\right.$ ). The conductive atomic force and scanning Kelvin probe microscopy experiments were conducted using a MFP-3D atomic force microscope (AFM) (NX-10, Park Systems) at the ambient environment in CAFM and SKPM mode, respectively. The Ir-based probe with Pt coating was EFM-10 produced by Nanoworld.

\section{Acknowledgements}

The research was supported by the "thousand talents" program for pioneer researchers and by the National Science Foundation of China (Grand No. 51572030), Beijing Natural Science Foundation (Grand No. 2162047), and Chinese Postdoctoral Science Foundation (Grand No. 2015 M 153816).

Received: ((will be filled in by the editorial staff))

Revised: ((will be filled in by the editorial staff)) Published online: ((will be filled in by the editorial staff))

[1] G. Chen, F. Abou-Galala, Z. Xu, B. M. Sadler, Opt. Express 16 (2008) 15059-15068.

[2] S. Kim, Y. T. Lim, E. G. Soltesz, A. M. De Grand, J. Lee, A. Nakayama, J. A. Parker, T. Mihaljevic, R. G. Laurence, D. M. Dor, L. H. Cohn, M. G. Bawendi, J. V. Frangioni, Nat. Biotechnol. 22 (2004) 93-97.

[3] H. Liu, N. Gao, M. Liao, X. Fang, Sci Rep. 5 (2015) 7716-1-9.

[4] X. Gong, M. Tong, Y. Xia, W. Cai, J. S. Moon, Y. Cao, G. Yu, C.-L. Shieh, B. Nilsson, A. J. Heeger, Science 325 (2009) 1665-1667.

[5] M. Ettenberg, Adv. Imaging 20 (2005) 29-32. 
[6] L. G. Hu, S. Dalgleish, M. M. Matsushita, H. Yoshikawa, K. Awaga, Nat. Commun. 5 (2014) 163-180.

[7] T. Zhai, L. Li , X. Wang, X. Fang, Y. Bando, D. Golberg, Adv. Funct. Mater. 20 (2010) 4233-4248.

[8] T. Choi, S. Lee, Y. J. Choi, V. Kiryukhin, S. W. Cheong, Science 324 (2009) 63-66.

[9] J. J. Zhang, X. D. Su, M. R. Shen, Z, H. Dai, L. J. Zhang, X. Y. He, W. X. Cheng, M. Y. Cao, G. F. Zou, Sci Rep. 3 (2013) 98-98.

[10] Z. L. Wang, Adv. Mater. 24 (2012) 4632-4646.

[11] Z. L. Wang, Nano Today 5(2010) 512-514.

[12] Y. B. Lee, J. Kwon , E. Hwang, C. H. Ra , W. J. Yoo, J. H. Ahn, J. H. Park, J. H. Cho, Adv. Mater. 27 (2015) 41-43.

[13] L. L. Xing, Y. F. Hu, P. L. Wang, Y. Y. Zhao, Y. X. Nie, P. Deng, X. Y. Xue, Appl. Phys. Lett. 104 (2014) 013109-1-6.

[14] B. Yu, Y. Fu, P. Wang, Y. Zhao, L. Xing, X. Xue, Phys. Chem. Chem. Phys. 17 (2015) 10856-10860.

[15] R. Macedo, F. A. Cardoso, S. Cardoso, P. P. Freitas, J. Germano, M. S. Piedade, Appl. Phys. Lett. 98 (2011) 1035031-3.

[16] L. Su, Z. X. Zhao, H. Y. Li, J. Yuan, Z. L. Wang, G. Z. Cao, and G. Zhu, ACS Nano 9 (2015) 11310-6.

[17] Z. X. Li, X. L. Liu, W. J. Chen, X. Y. Zhang, Ying Wang, W. M. Xiong, Yue Zheng, AIP Advances 4 (2014) 127111-1-10.

[18] D. F. Pan, G. F. Bi, G. Y. Chen, H. Zhang, J. M. Liu, G. H. Wang, J. G. Wan, Sci Rep. 6 (2016) 22948-1-9.

[19] J. Seidel, D. Fu, S. Y. Yang, E. Alarcón-Lladó, J. Wu, R. Ramesh, J. W. Ager, Phys. Rev. Lett. 107 (2011) 1247-1250.

[20] Y. M. Sheu, S. A. Trugman, Y.-S. Park, S. Lee, H. T. Yi, S.-W. Cheong, Q. X. Jia, A. 
J. Taylor, R. P. Prasankumar, Appl. Phys. Lett. 100 (2012) 4707-4715.

[21] K. Wasa, T. Matsushima, H. Adachi, T. Matsunaga, T. Yanagitani, T. Yamamoto, J. Appl. Phys. 117 (2015) 124106-1-8.

[22] L. Pintilie, I. Vrejoiu, G. Le Rhun, M. Alexe, J. Appl. Phys. 101 (2007) 064109-1-8.

[23] B. Chen, Z. H. Zuo, Y. W. Liu, Q. F. Zhan, Y. L. Xie, H. L. Yang, G. H. Dai, Z. X. Li, G. J. Xu, R. W. Li, Appl. Phys. Lett. 100 (2012) 173903-1-4.

[24] L. Pintilie, M. Alexe, A. Pignolet, D. Hesse, Appl. Phys. Lett. 73 (1998) 342-344.

[25] M. Noda, H. Zhu, H. Xu, T. Mukaigawa, K. Hashimoto, T. Kiyomoto, R. Kubo, H. Tanaka, T.Usuki, M. Okuyama, Integrated ferroelectrics, 35 (2001) 31-39.

[26] S. Kumari, N. Ortega, D. K. Pradhan, A. Kumar, J. F. Scott, R. S. Katiyar, J. Appl. Phys. 118 (2015) 184103-1-10.

[27] S. M. Niu, S. H. Wang, L. Lin, Y. Liu, Y. S. Zhou, Y. F. Hua, Z. L. Wang, Energy Environ. Sci. 6 (2013) 3576-3583.

[28] G. Zhu, J. Chen, Y. Liu, P. Bai, Y. S. Zhou, Q. S. Jing, C. F. Pan, Z. L. Wang, Nano Lett. 13 (2013) 2282-2289.

[29] X. Y. Wei, G. Zhu, Z. L. Wang, Nano Energy 10 (2014) 83-89.

[30] J. Xing, E. J. Guo, J. J. Dong, H. Y. Hao, Z. Y. Zheng, C. C. Zhao, Appl. Phys. Lett. 106 (2015) 033504-1-5.

[31] I. Vrejoiu, G. Le Rhun, M. Alexe, J. Appl. Phys. 101 (2007) 064109-1-8.

[32] S. Sharma, M. Tomar, N. K. Puri, V. Gupta, Sensors and Actuators A 230 (2015) 175181.

[33] A. Zomorrodian, N. J. Wu, Y. Song, S. Stahl, A. Ignatiev, E. B. Trexler, C. A. Garcia, Jpn. J. Appl. Phys. Pt. 1. 44 (2005) 6105-6108.

[34] Y. S. Zhou, Y. Liu, G. Zhu, Z. H. Lin, C. F. Pan, Q. S. Jing, Z. L. Wang, Nano Lett. 13 (2013) 2771-2776. 
[34] D. L. Shao, J. Gao, P. Chow, H. T. Sun, G. Q. Xin, P. Sharma, J. Lian, N. A. Koratkar, S. Sawyer, Nano Lett. 15 (2015) 3787-3792.

[35] J. A. Rodriguez, A. Etxeberria, L. González, A. Maiti, Chem. Phys. 117 (2002) 26992709.

[36] C. Li, Y. Ding, M. Soliman, J. Lorenzo, N. Dhasmana, P. Chantharasupawong, A. V. levlev, A. J. Gesquiere, L. Tetard, J. Thomas, ACS Appl. Mat. Interfaces 8 (2016) 4730-4738. 

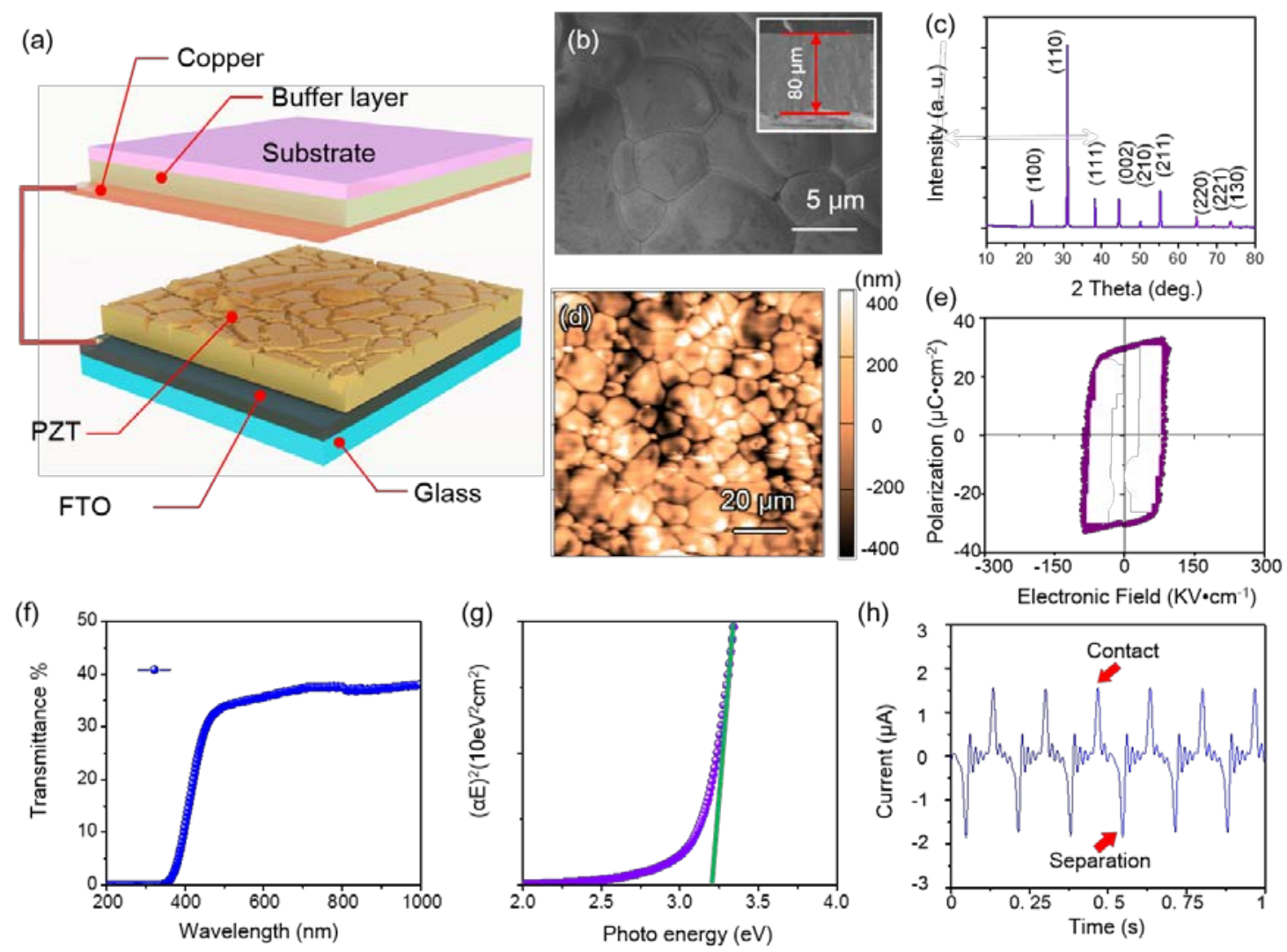

Fig. 1. Structure and characterization of a UV-SPPD. (a) The device structure of a UV-SPPD. (b) Top and side view (insets) SEM images of the PZT film. (c) XRD diffraction pattern and (d) morphological roughness of the PZT film measured by AFM. (e) P-E hysteresis loops and (f) transmission spectrum of the PZT film. (g) Plot of $(\alpha E)^{2}$ versus $E$ for the PZT film near the optical band gap and linear extrapolation to determine the optical band gap. (h) Short-circuit current $\left(I_{\mathrm{sc}}\right)$ output of the UV-SPPD without illumination. 

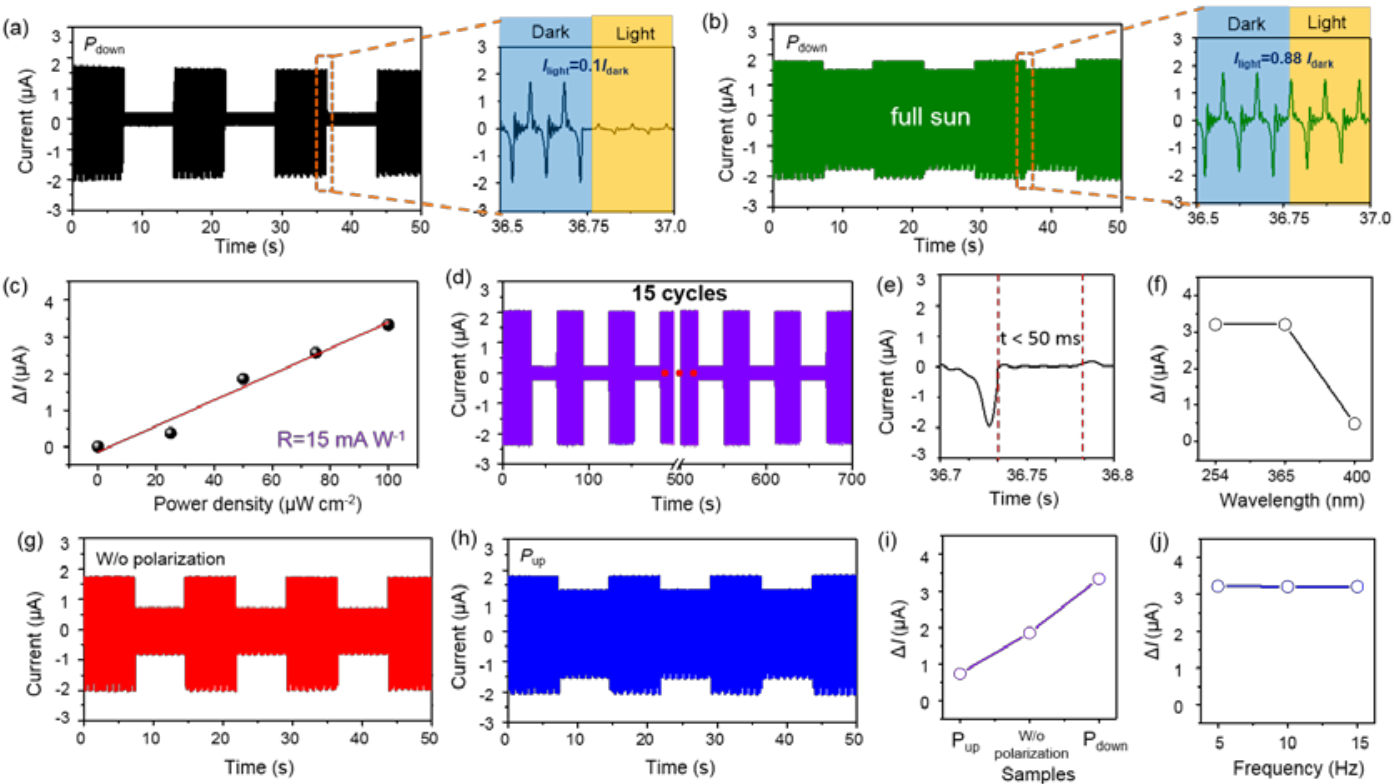

Fig. 2. Change of the $I_{\mathrm{sc}}$ of the UV-SPPD. (a) $P_{\text {down }}$ type under $5 \mathrm{~Hz}$ mechanical vibration frequency under UV illumination (365 nm, $100 \mu \mathrm{W} \mathrm{cm} \mathrm{cm}^{-2}$ ) (b) and under full illumination. (c) Dependence of the values of $\Delta I\left(I_{\text {dark }}-I_{\text {light }}\right)$ under various UV light power density and its linear fitting result. (d) Photocurrent response during 15 cycles. (e) The response time of the current. (f) Photocurrent spectral response of the UV-SPPD, and all the photocurrent were measured at $100 \mu \mathrm{W} \mathrm{cm}{ }^{-2}$. (g) Without polarization and (h) $P_{\text {up }}$ type under UV illumination with $5 \mathrm{~Hz}$ mechanical vibration frequency. (i) The $\Delta I$ of different types of UV-SPPD. (j) The $\Delta I$ of the $P_{\text {down }}$ type UV-SPPD under different vibration frequencies. 


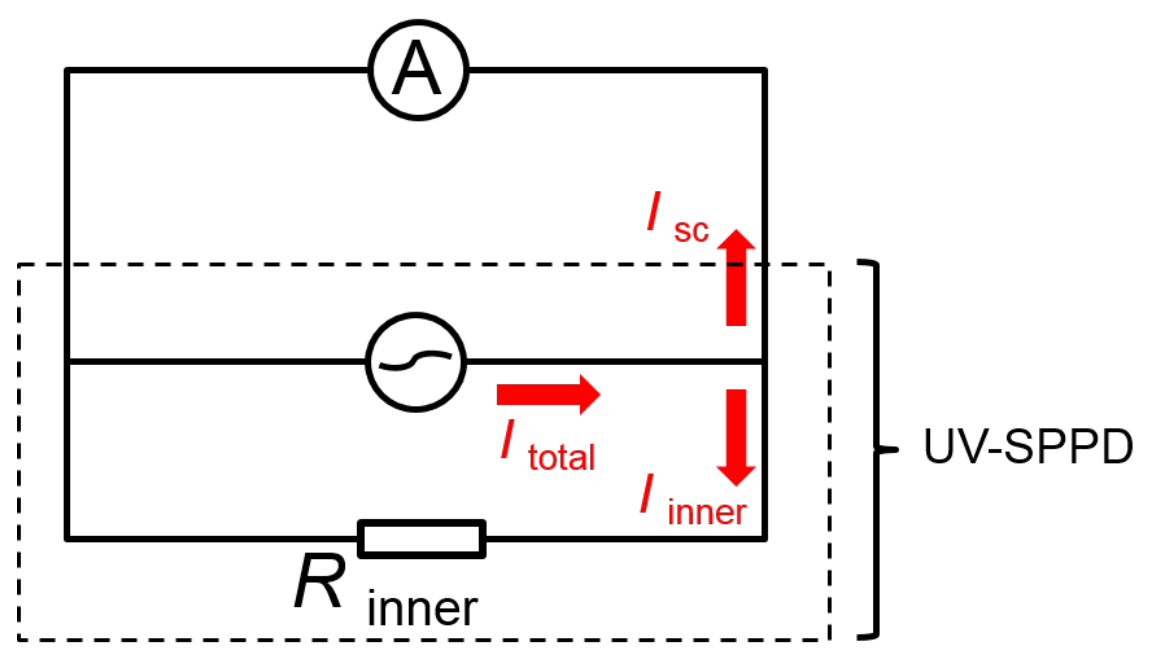

Fig. 3. Schematic that illustrates the circuits to the output current from the UV-SPPD. The whole circuit was divided into $I_{\text {total }}, I_{\mathrm{sc}}$ and $I_{\text {inner, }}$, which represent the total current generated by the circuit, the measured short-circuit output current, and the internal current of the electric source, respectively. 


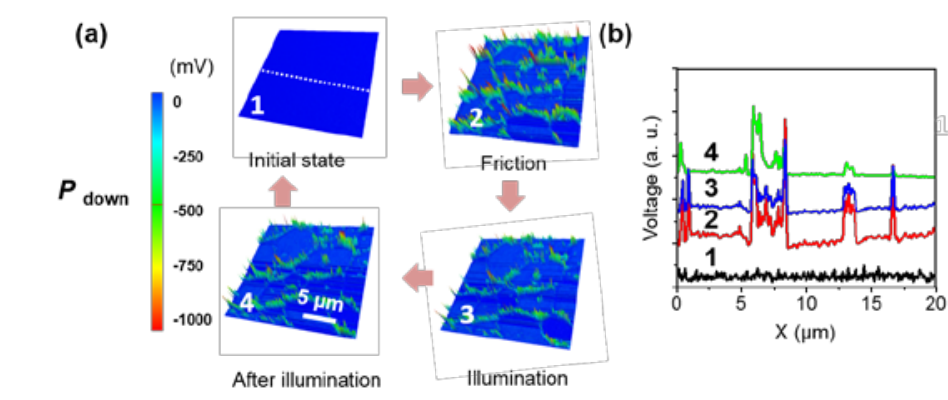

(c)

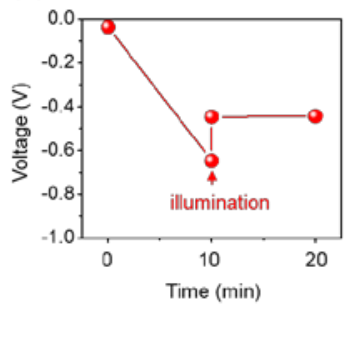

(g)
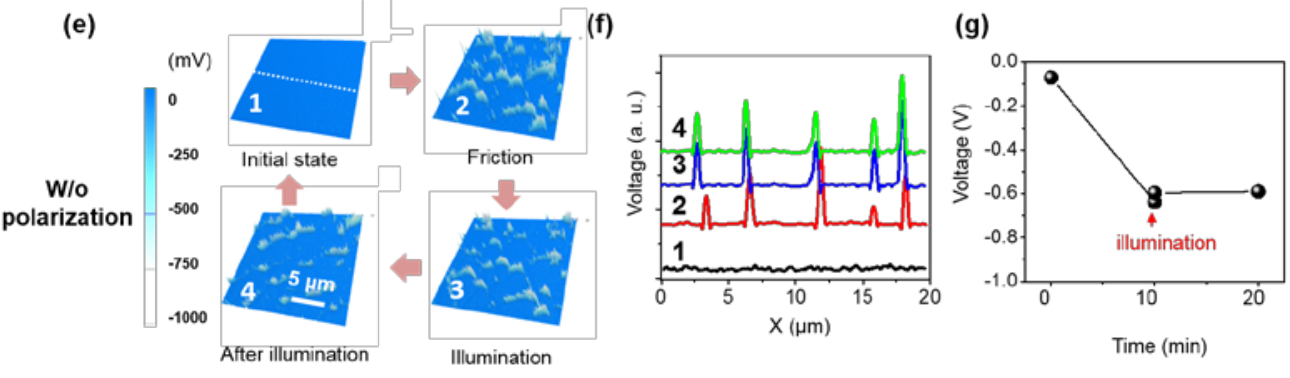

(h)
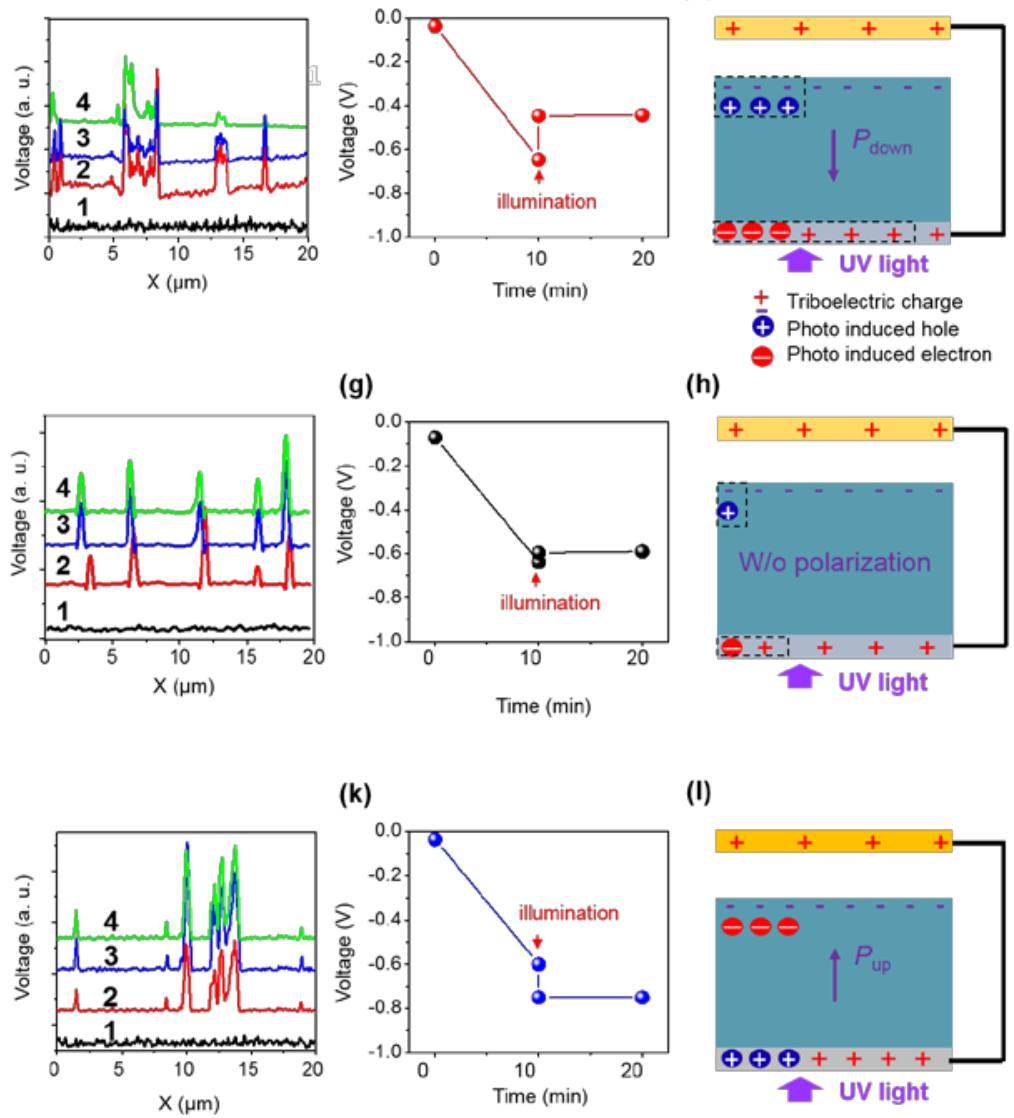

(k)

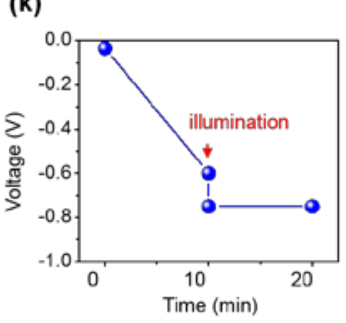

(I)

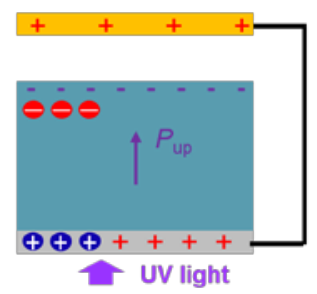

Fig. 4. Characterization of the surface potential upon illumination. $3 \mathrm{D}$ mappings of the surface potential after friction and with illumination applied 10 min after the friction: (a) $P$ down, (e) without polarization, and (i) $P_{\text {up }}$ PZT films. Cross-sectional profile of the potential distribution: (b) $P_{\text {down }}$ (f) without polarization, and (j) $P_{\text {up }}$ PZT films. Corresponding values of the surface potential as a function of time: (c) $P_{\text {down }}$, (g) without polarization, and (k) $P_{\text {up }}$ PZT films. Schematic of the charge transfer of the UV-SPPD: (d) without polarization, (h) $P_{\text {down }}$, and (i) $P_{\text {up }}$ PZT film. 

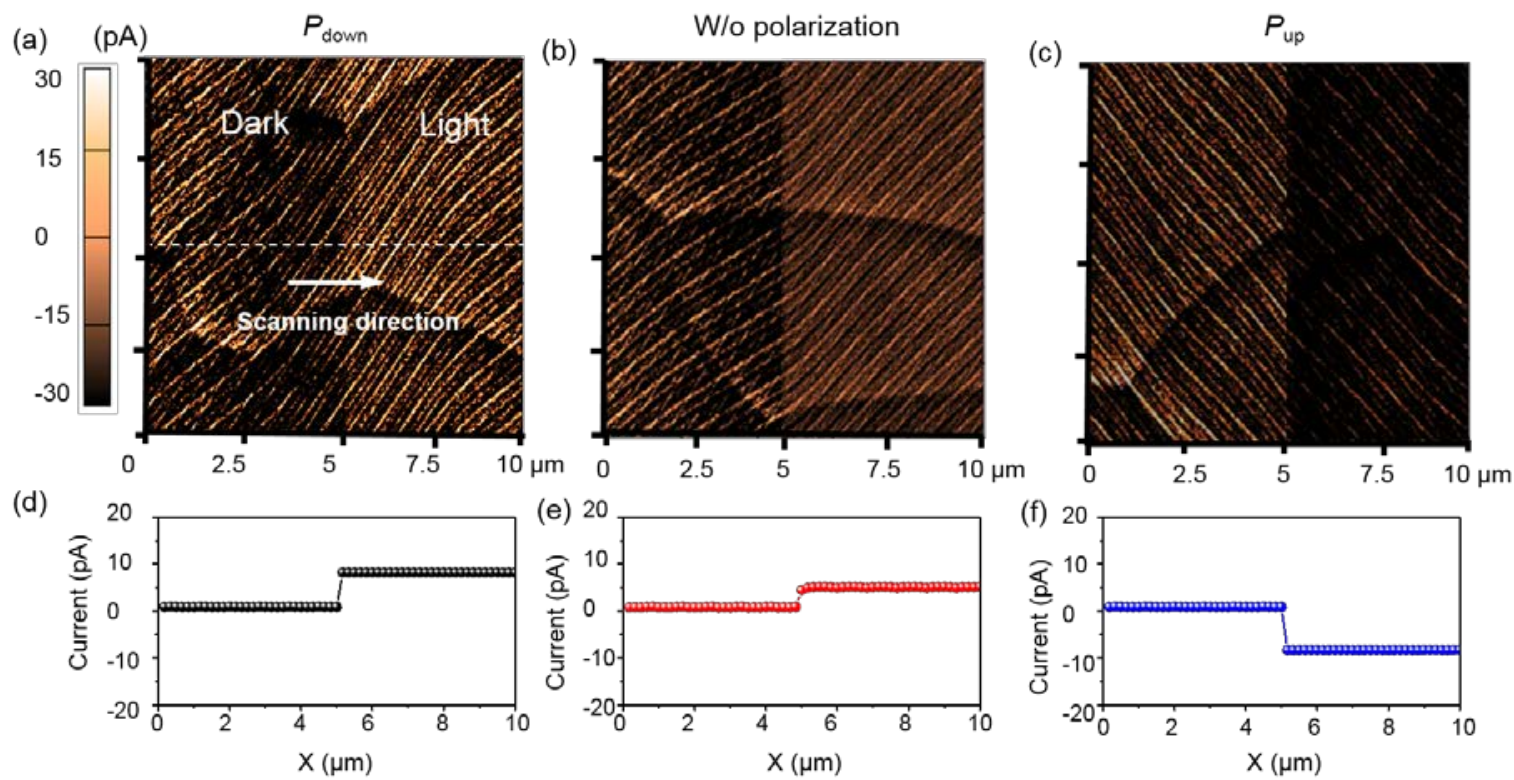

Fig. 5. Characterization of thin-film conductivity upon illumination. Current mapping with or without illumination at zero bias: (a) $P_{\text {down }}$, (b) without polarization, and (c) $P_{\text {up }}$ PZT film. Corresponding cross-sectional profile of the current distribution (d, e, and f). 
A UV self-powered photodetector (UV-SPPD) that relies on the joint properties of the photoelectric effect and the triboelectric effect of a PZT film was fabricated and characterized. A high UV detecting performance was obtained. A two-fold sensing mechanism based on the variation of the surface charge density and the conductivity was proposed to explain the working mechanism.

Keyword: Lead zirconium titanate, Polarization, Self-powered, Triboelectric effect, UV photodetector

Li Su ${ }^{1}$, Hua Yang $\mathrm{Li}^{1}$, Ying Wang ${ }^{1}$, Shuang Yang Kuang ${ }^{1}$, Zhong Lin Wang*1, ${ }^{1}$, Guang Zhu*1

Coupling of Photoelectric and Triboelectric Effects as an Effective Approach for PZTBased High-Performance Self-Powered Ultraviolet Photodetector

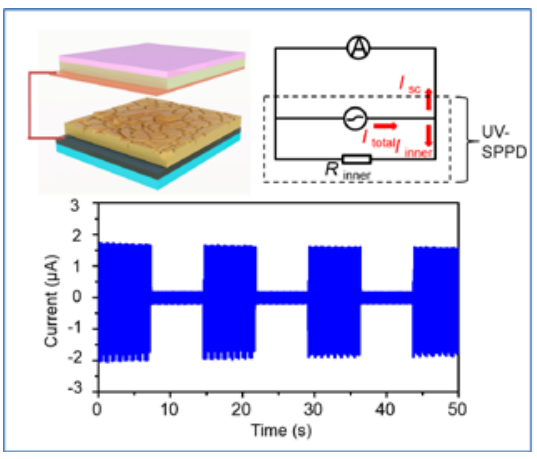

